\title{
Implementation of QT Interval Measurement to Remove Errors in ECG
}

\author{
S. Chitra \\ Research Scholar \\ School of Computing Sciences \\ VISTAS, Chennai, India
}

\author{
Dr. V. Jayalakshmi \\ Professor \\ Department of Computer Applications \\ VISTAS, Chennai, India
}

\begin{abstract}
Wireless Body Sensor Network (WBSNs) are devices that can be ported with different detection, storage, computer, but also communication capabilities. Interfacing was beneficial whenever information was collected by many sources, which may lead to erroneous sensory information. During this paper, an information nuclear fission Ensembles technique for working raw healthcare information through WBSNs during ambient cloud computer settings as described. Monitoring data were collected through various instruments and combined to provide statistics on high movements. The simulation was conducted using the low-cost Internet of Things (IoT) surveillance system on chronic kidney disease (CKD). Biosensors have been used in healthcare surveillance systems to record health problems. Patients with CKD would benefit from the developed surveillance system, which will facilitate the early diagnosis of the predominant diseases. This merged information was then sent into using the Aggregation algorithm can forecast premature cardiac illness and CKD. These groups were housed within a Cloud processing context; therefore these forecasting calculations were distributed. Another lengthy practical investigation backs that system provides application, while those findings were encouraging, with 98 percent efficiency whenever the height of that tree was equivalent with 15 , total amount if estimation methods are 40, while the overall predicting job was based upon 8 attributes. We compute a mean square ECG waveform from all available leads and use a new technique to measure the QT interval. We tested this algorithm using standard and unique ECG databases. Our real-time QT interval measurement algorithm was found to be stable, accurate, and capable of tracking changing QT values.
\end{abstract} IoT

Keywords-e-Health; $Q T$ interval; GPU; ECG signal; CKD;

\section{INTRODUCTION}

In various ways, clinical wearing gadgets differ from overall technologies. Consumer engagement is often far very restricted. The volume of its CPU, information memory, \& energy endurance is typically used to make an efficient market [1]. Signals analysis was emphasized for this product at the level which was rarely seen in overall worn computer technologies. Ultimately, on aspects such as confidentiality, dependability, governmental laws, \& company's lawful obligation, wearing clinical systems must meet higher standards [2]. Researchers shall concentrate upon clinical wearing gadgets throughout the study. Wireless Body Sensor networks (WBSNs) were changing the face of medical technologies [3]. Worn gadgets having devices that could identify physiologic indicators, portals that facilitate internet connection, \& back- and company front computers in storing, analyzing, \& presenting data were all part of such systems [46]. WBSN\& smart gadget technologies aren't quite innovative.

Such transportable monitoring gadgets can help throughout their treatment monitoring chronic illnesses like cardiovascular assaults, bronchitis, and hypertension, as much as enabling monitoring collecting vital facts about such patient's individual wearer's anatomy. Electrocardiogram, blood oxygenation saturation, breathing, \& physical obesity may all be monitored pulses [7]. The clinical assist gadget was intended to give lengthy help to disabled people. Regarding individuals undergoing rehabilitative, the portable recovery support gadget integrates surveillance \& healthcare support capabilities [8]. This monitoring function improved that child's recuperation by allowing them to escape potentially dangerous situations including risks. Furthermore, throughout recovery, this healthcare assistance gadget may assist in transitional limitations.

Its portable product's detectors monitor physiologic signals of its physique [9]. Non-invasive, dependable, small, portable, changeable, \& ready to integrate into a gadget are all desirable qualities. Its monitor's selection is dependent upon its intended usage [10]. When its intended purpose was the cardiac tracking device, its device's characteristics, like sensitivity and sample frequency, must be determined [11]. Next researchers must pick any of those detectors which best fit our needs.

The highlights of this work are as follows:

1) Data from various sensors is combined to provide higher-quality data that is fed into ensembles for heart disease and CKD prediction.

2) The ensembles are placed in a fog computing environment, and the separate forecasters' findings were integrated into providing an overall cohesive outcome.

3) Within such cloud computer context, researchers explore using innovative kernels randomized woodland towards its forecasting problem. Exponential randomized forests have been found to significantly outperform commonly used compositions throughout terms of forecasting ability. 


\section{LITERATURE SURVEY}

The portable gadget, by speaking, offers restricted energy $\&$ computing performance. Because of this constraint, the choice must be made on both types \& quantities of information that should be collected, saved, \& communicated. A portable healthcare equipment's primary goals were able to assist physicians or caretakers via delivering relevant data about the client's condition [12]. This would neither suggest that ascending sensory material must be ignored; rather, basic information must be modified to give better valuable facts in judgment [13]. As this result, researchers must examine which data should be sent to a physician or caretaker, as well as whether to analyze this to receive valuable data. Among the most important technology for wearing healthcare equipment was communications. Connectivity among sensing boards \& back-end systems, as well as the linkage among a back-end scheme \& its medical practitioner, are often involved [14]. There exists a standardization problem for considering when it comes to device-to-device compatibility.

The primary purpose of such a sensing layer was acquiring physiologic data on this target [15]. Wireless and cable connections would be being used to send the information beyond an information layer, where they would either be saved or exchanged. On this applications layer, these impulses were examined \& recreated as treated information. Instruments in any surveillance system were often connected to the destination, whether could be either a physician or the gadget operator [16]. Sensor's elements, a device with cameras pretreatment like is filtration \& compression, \& communications among both sensors \& information plane are all included within a monitors sector [17]. The instrument parts were generally chosen based on the anticipated application. This sensor element must detect its glycemic levels when its doctor wishes to track a concentration of sugar within the patients.

Because of many sensors the shortage of resources substantial computing skills, very basic pretreatment methods like filtration \& compression were commonly included. Processing could make a gadget better personality \& functional particularly while its networking connectivity was interrupted, however, it must be done with at least quantity of information deformation possible [18]. To communicate basic information for an information plane sensing layer, each sensing plane typically includes a minimum single connected and wirelessly communications link. Challenges upon that sensing plane include a sensing vehicle's lifespan \& physical architecture, customer acceptance, confidentiality, \& safety issues [19]. This sensing plane's key characteristics were immediately tied with a sensing gadget. Every sensing gadget must assess when long it will continue to gather its data. Whenever a detector is to detect movement, this must be triggered whenever that movement happens. When a sensor is required to capture ECG data, it must be operational over the length when duration.

This information plane serves for a system bridging even though that was placed among its sensing planes \& both applications layers. Although that sensing plane and applications planes deal with precise focused information, its information planes must avoid being too generic [20]. This must contain all appropriate connections that facilitate gathering information via many sensing units. Any program might be required to merge and recycle information as the result of a constraint. Because of its sensitivity to physical information, it is necessary must offer such connections proper protection of information accessibility [21]. Because an information plane has 2 routes of transferring information, one is sensing planes and another for the applications planes, all regulations on obtaining information across every platform must be distinct.

Generic networking such as the internet or mobile networking such as GSM, UMTS, CDMA, \& WiMAX is used to communicate between an information plane towards the applications planes. An applications layer, by broad, arranges their unique approach of presenting, viewing, \& analyzing information across a generalized substrate like computers, tablets, or cellphones [22]. As a result, information interchange becomes a principal source of contact among information planes \& an applications layer. Just at software planes, these were two types of information sharing methodologies: sealed information swapping \& accessible information swapping. Holding apps often employ connections internet technology can transport data into another informational level.

\section{PROPOSED METHOD}

The echocardiogram was used to examine such electrical motion within the heart (ECG). This detects electronic signals produced from heart muscle polarization \& relaxation \& converts them to a waveform. This is another critical tool for cardiovascular research \& diagnostics. A P waveform, a QRS complicated, a T tidal, and a U generate buzzing swarming up a normal ECG trace of a heart cycle. The $\mathrm{P} \& \mathrm{~T}$ phases typically exceed this $U$ waveform, which was typically undetectable. This Interphase was my attention since it has proven proved to be an excellent predictor of existence cardiovascular problems. A timing differential from commencement to that QRS or a conclusion with a $\mathrm{T}$ wave was used to calculate it. Signals separation was required to estimate the QT duration, however, that was difficult owing to the peculiarities of ECG constituent waveforms. A QT duration must be adjusted when examining QT readings across the period at varying cardiac rates because it fluctuates on cardiac speed. In generally, Bazzett's equation (1) was commonly employed to calculate an adjusted QT duration, abbreviated QTC:

$Q T^{c}=\frac{\|Q T\|}{\sqrt{\|R R\|}}$

wherein $|R R|$ denotes the duration of the R-R intervals across its present $\mathrm{R}$ maximum \& its preceding $\mathrm{R}$ high, \& $|\mathrm{QT}|$ denotes the distance at that start of $\mathrm{Q} \&$ that conclusion of $\mathrm{T}$. To track the QTC upon a beat-by-beat basis, researchers must separate all calibration events for each pulse, including for its QRS start, $\mathrm{T}$ discrepancy, \& $\mathrm{R}$ maximum. This proposed approach demonstrates an efficient approach that divides every calibration spot \& computes QTC enabling heart rate assessment shown in Fig. 1. 


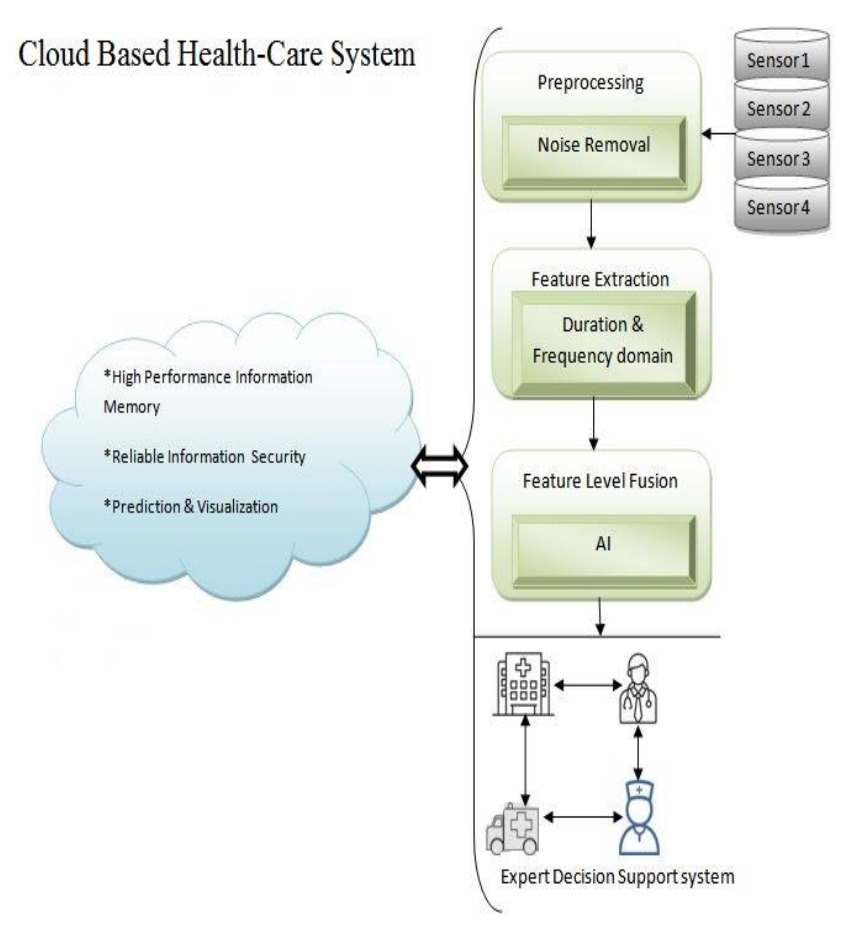

Fig. 1. Flow Diagram Cloud-Based Health Care System.

Researchers require a previous understanding of underlying structures in relevance for locating calibration sites given any series of data. Such was referred to as prototypes and they were standard structures that researchers were looking for. Creating the framework using numerous streams is proposed within this technique. Si denotes the ith individual sample from the $\mathrm{i}^{\text {th }}$ channel (j). $\mathrm{T}$ is a pattern that may be described by

$T_{s}=\left|T_{1}, T_{2}, \ldots, T_{n}\right|$

$T_{x}=\mid S_{x}(y-h), S_{x}(y-h+1), \ldots \ldots, S_{x}(y), \ldots \ldots, S_{x}(y+$

h)| $\left.\right|^{T}$

when $\mathrm{n}$ denotes the number of networks employed throughout this study $\& 2 \mathrm{~h}+1$ denotes the overall duration of a proposed system. To reduce the influence of disturbance, researchers use the Gaussian functional to create company center balanced masks. Where $U_{x}$ be the $1 \mathrm{D}$ Gauss weighting vectors of length $2 \mathrm{k}+1$ for such ith channels.

$(n)=\exp \left(-\frac{1}{2}\left(\frac{\left|i^{2}-h^{2}\right|}{\partial^{2}}\right)\right)$

$U_{x}=\left|h_{1}, h_{2}, \ldots h_{2}\right|^{T}$

The Gaussian value $\mathrm{U}$ can be defined as

$U=\left|U_{1}, U_{2}, \ldots, U_{n}\right|$

Where $\mathrm{U}_{1}=\mathrm{U}_{2}={ }^{\cdots}=\mathrm{G}_{\mathrm{n}}$. The final template $\mathrm{T}$ author use in the analysis can be defined as

$T=\operatorname{Mask}\left(U, T_{s}\right)$

where, Mask $(\mathrm{E}, \mathrm{F})$ returns the elements-wise product of $\mathrm{E}$ $\& \mathrm{~F}$.

\section{RESULT AND DISCUSSION}

Several sources like disturbance could readily compromise ECG readings. This preparation stage's goal was to filter out interference which is not as legitimate ECG data. One of those most prevalent ECG data the foundations started sliding due to irregularities. Organization drifting \& higher frequencies noisy artifacts could be reduced using band-pass filtration. Because that bulk of ECG impulses were found as in inferior range below $100 \mathrm{~Hz}$, our proposed approach uses the bandpass filters having cut-off wavelengths between $0.5 \mathrm{~Hz} \& 100$ $\mathrm{Hz}$. Another pseudo-periodic output includes an ECG pulse. Each pulse must be segmented to a beat-by-beat assessment. This QRS Sequence was the most prominent output of every ECG rhythm. It might serve as a useful divider between neighboring rhythms. Each calibration position must be determined among both earlier \& later R spikes, \& its R-R separation must be calculated using Bazzett's algorithm to determine an adjusted QT period. Because of that, every QT research requires locating the QRS complicated.

Researchers currently know the location of every calibration site, its commencement of such QRS, \& its dispersion of $T$ waves. Such structures were depicted within these designs, which might clarify those fiducially locations in the middle of all those frameworks. Our proposed approach identifies its most matched site regarding the provided pattern during a detecting stage. This was just a single matched spot in such a beating data that was a signal across successive $R$ spikes, according to a property in this ECG sensor. During heart rate assessment, our proposed approach uses the outcome of a high point sensing phase as just a beating divider. Where $\mathrm{R}=\{\mathrm{r} 1, \mathrm{r} 2, \ldots, \mathrm{rn}\}$ represent a maximum identification result. When addressing any boundaries within the proposed approach, the enlarged signals would be being used up towards the duration of such a specified template.

$$
\begin{aligned}
& E_{x}=\left|S_{x}\left(r_{h}-\frac{z}{2}\right), S_{x}\left(r_{h}-\frac{z}{2}+1\right), \ldots, S_{x}\left(r_{h+1}+\frac{z}{2}\right)\right|^{T} \\
& E=\left|E_{1}, E_{2}, \ldots, E_{n}\right|
\end{aligned}
$$

Whereas $n$ specifies this same number many channels will get studied and w indicates its pattern length.

To get that greatest fitting place when matched to the particular pattern, researchers must evaluate the overall closeness of every place towards the supplied framework. The approach uses a Pearson correlations value, which is described as the measure of the closeness among such signals \& the particular framework shown in Fig. 2.

$r_{i j}=\frac{\sum_{x=1}^{m}\left(I_{x}-\bar{I}\right)\left(J_{x}-\bar{J}\right)}{\sqrt{\sum_{x=1}^{m}\left(I_{x}^{2}-\bar{I}^{2}\right)} \sqrt{\sum_{x=1}^{m}\left(J_{x}^{2}-\bar{J}^{2}\right)}}$

That slicing signals Bs for such a specific position $\mathrm{k}$, that is the identical lengths as any templates, may be described by

$$
\begin{aligned}
& E_{x, s}=\left|E_{x}(h), E_{x}(h+1), \ldots, E_{x}(h+z-1)\right|^{T} \\
& E_{s}=\left|E_{1, s}, E_{2, s}, \ldots, E_{n, s}\right|
\end{aligned}
$$

when, $\mathrm{w}$ is that width for a specified pattern \& $\mathrm{n}$ denotes the number of streams under consideration. Every one of those indicators would be explored by computing Pearson terms of 
interaction using company particular templates following component sequential multiplying using the Gaussian masking, as specified by Eq. (11).

\section{A. Decision Rule}

Researchers must explore those locations which match all requirements connected towards the features to validate the overall authenticity of a sought fiducial location. Researchers currently get knowledge upon that broad-spectrum between periods based upon its limits on the individual signals. These next checks are used in this technique to demonstrate the integrity of a supplied collection in Equation (13).

$Q T_{c}^{x}=\left|r_{x}, r_{x+1}, Q_{o n}(x-1), T_{o f f}(x)\right|$

Researchers could derive ith adjusted QT intervals from one pair of QTic.

Several people have a heartbeat with fewer than 220 beats per minute. The usual limit of an adult's heartbeats is $60-100$ beats per minute. Youth individuals, on the other hand, had quicker heartbeats than elders. A spectrum in adult heartbeats is depicted on this page. Our proposed method limits your pulse rhythm spectrum between $40-200$ beats per minute. The offset must be found before Qset for a one beats output. This proposed approach excludes all items submitted in evaluation if a condition was never satisfied with this provided data. Several studies had attempted to demonstrate a rectified QT interval's potential length. This spectrum must remain around $0.3 \& 0.6$, according to experiments. For being proven like a legitimate database, any submitted information source should likewise fulfill these conditions.

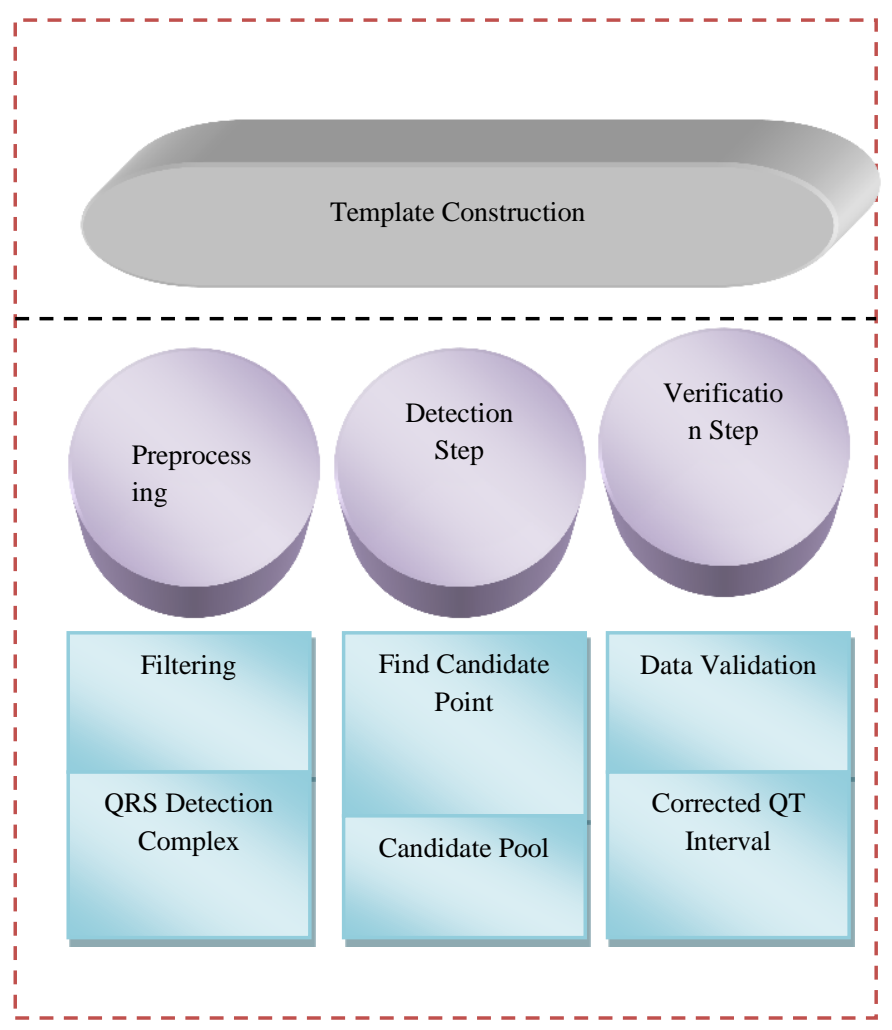

Fig. 2. The Proposed Subsystem Approach.

\section{B. Testing}

Researchers provide my method for implementing the recommended method. This architecture for the technology is depicted in Fig. 3. This technology was divided into two sections: service and user. Because this was an internet program, its user sides were built up using web interactions that communicate through HTTP.

A Global Standards of Healthcare Photos \& Associated Data was Computerized Imagery \& Telecommunications for Health. This specifies codecs of healthcare pictures X-rays, CT examinations, mammograms, and geomagnetic receptor tomography are among examples (MRI) that may be shared while maintaining all information \& clarity required for therapeutic application. Frequency information, inspired are electrocardiographic \& circulatory information, could be altered within the DICOM standard, as per the DICOM supplemental. The DICOM translator was the data center application. Researchers implement business DICOM standards on my program to allow customers can manipulate electrocardiographic data for sources towards a computer while maintaining compliance using records of commonly employed recording equipment.

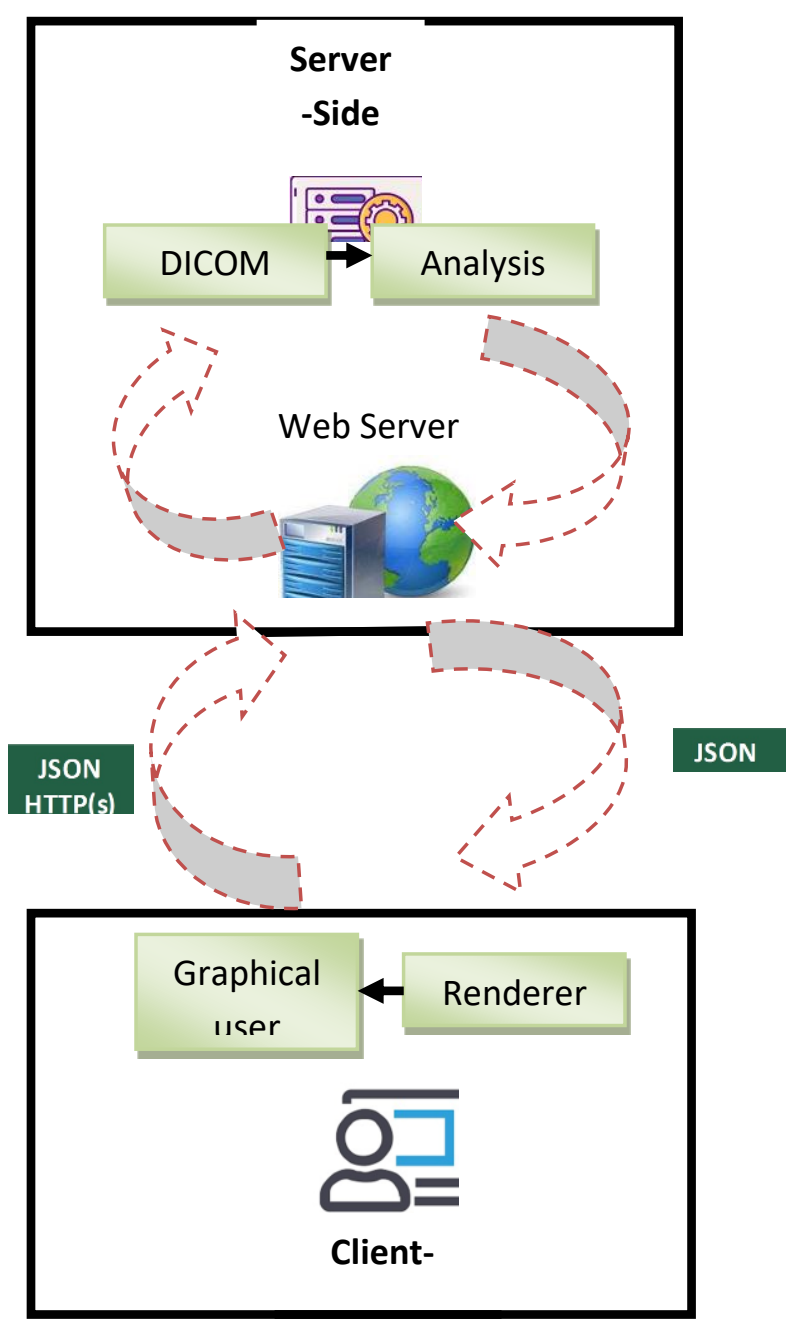

Fig. 3. Design of the Webserver of QT Research. 
As per Fig. 2, this information was prepared as DICOM on the user end and later communicated towards the service end via GUI. Because JSON via HTTP was straightforward \& quick to deploy, researchers utilize this as an information exchange mechanism. All transferred information was converted into amounts only at the DICOM decoder via interpreting information supplied within the DICOM standard. That digitized output may be used as an output for the proposed method in 5.2, which was performed within the research engines' service element. Some findings of an operation were removed towards such internet host, which converts these into JSON-formatted information \& sends this to a user. Researchers used python $2.7 \&$ the Tornadoes internet browser here on the service end, \&angularjs\& HTML5 just on the consumer edge.

Upon that consumer end, we'll have to create multiple graphical customer interface webpages to interact with a customer $\&$ view inputs $\&$ process information.

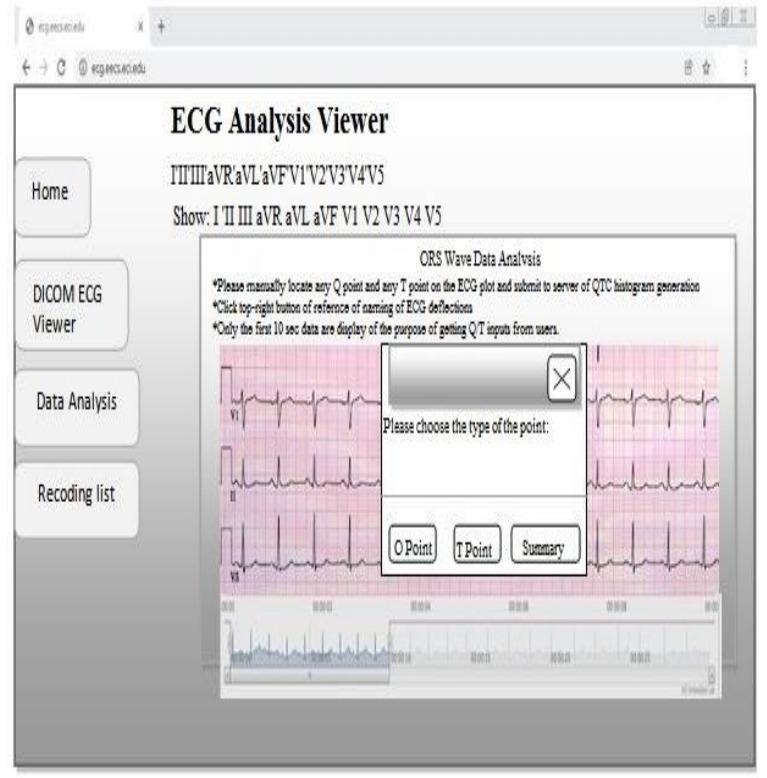

Fig. 4. Selecting of Fiducially Elements in Creating Patterns there in GUI.

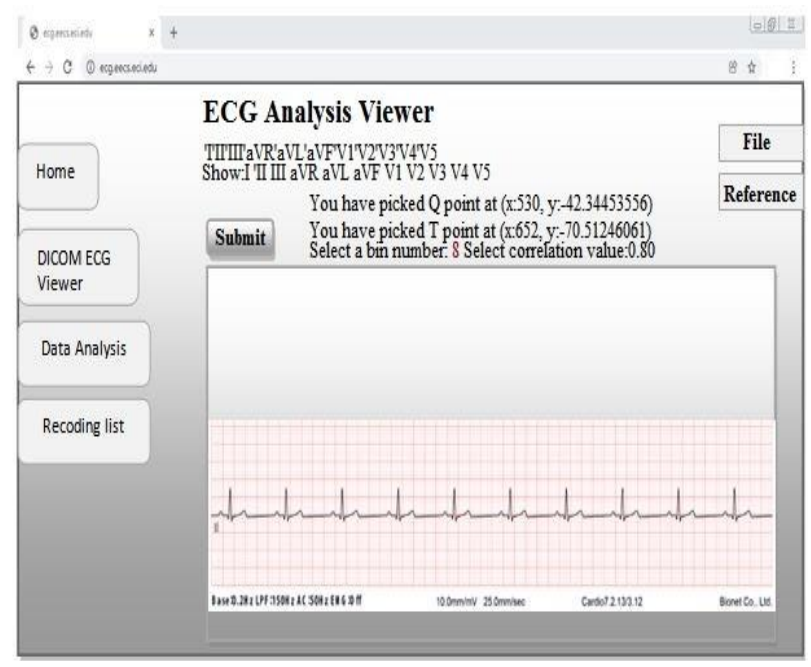

Fig. 5. Initializing the Attributes.
This GUI interface for selecting fiducial locations is shown in Fig. 4. These equations of QRS start \& T Distortion of the surges were dynamically developed thanks to customer input here on location. Researchers must establish starting settings as shown in Fig. 5 following constructing algorithms of looking to every fiducial location. These $\mathrm{R}$ spikes are found using individual streams using a proposed technique. As a result, consumers can select so that channels are covered for peak monitoring. An additional variable, thresholds, was a minimal number that must be fulfilled for modeling \& indicators can remain comparable. When the overall crossing metric rating for relationships of the potential fiducial spots is lower over a criterion, the proposed method excludes them within the evaluation. A quantity of more bits of such QT measurement results was likewise determined. QT assessment outcome would be shown on your GUI as a histogram-like dispersion.

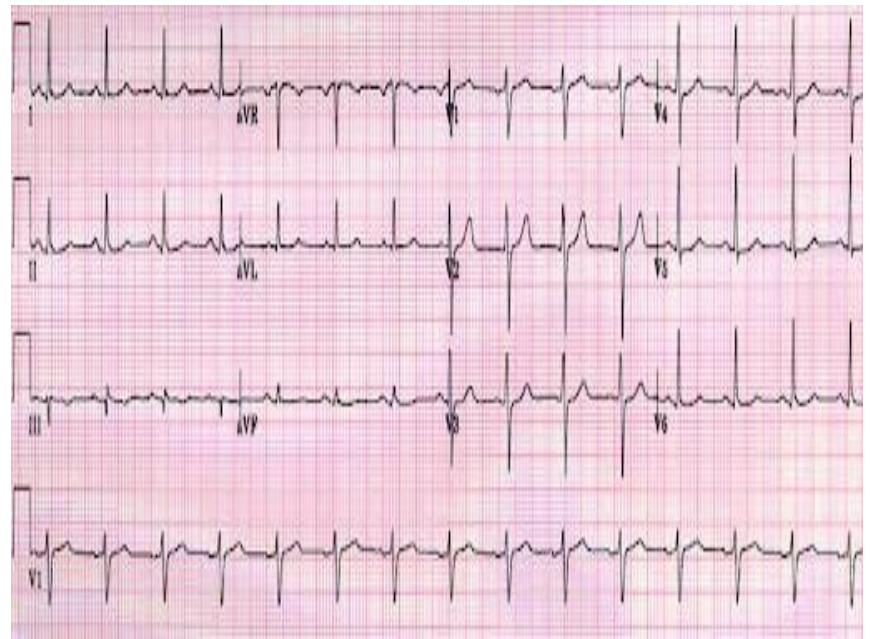

Fig. 6. Image of an ECG in the Typical ECG Style.

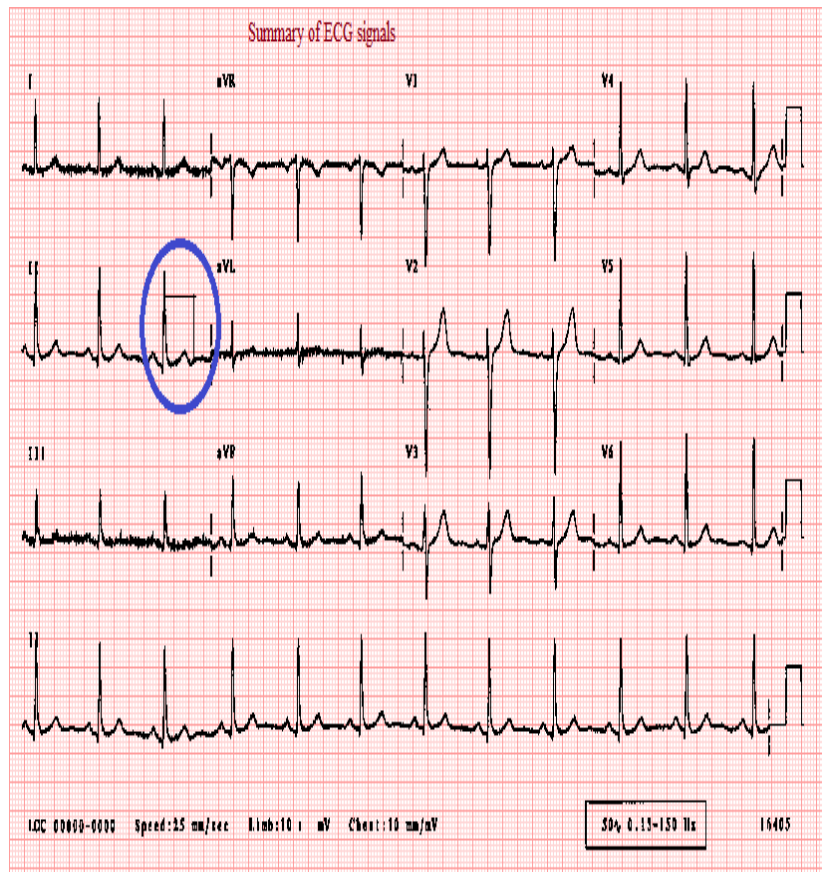

Fig. 7. Using the Caliper Tool to measure the duration of Heart Disease. 
Its user interface additionally includes functionalities that resemble a conventional style in aiding physicians in their analysis. Fig. 6 shows how the information was displayed in a real-world sheet style. Caliper capability is incorporated on a GUI webpage to give a straightforward technique of measuring periods to manually evaluate through physicians. Another purple arrow from Fig. 7 and 8 represents the separation measurements findings from two separate places over each transmission for CKD and heart disease.

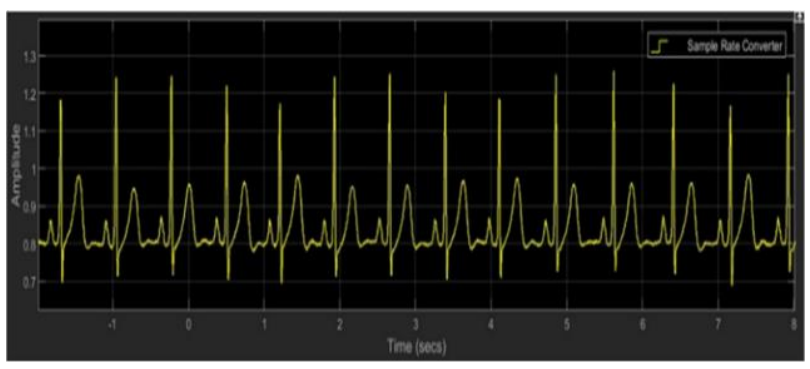

Fig. 8. Using the Caliper Tool to measure duration CKD.

\section{CONCLUSION}

The QT interval was a crucial statistic that may be used as a reliable indicator for cardiac illness. However, owing to the characteristics of ECG transmitter constituent waveforms, its nature of assessments, its absence of standards, \& its huge quantity of information, this was difficult to evaluate autonomously. During beat-by-beat QT assessment, researchers proposed a novel method. Researchers also used the proposed technique to create an internet solution for QT assessment. In addition, the software included several essential features for instructions and automated QT evaluation. Researchers used the DICOM recorded standard to ensure interoperability that using existing recording equipment. To determine the heart rate, the attenuated ECG signals were analyzed for CKD and heart disease through Q wave spectral analysis. The displayed heart rate was well below the heart rate range of 79-82 beats per minute (bpm). The $\mathrm{Q}$ wave found was also similar to those of the QT algorithm. The filter ECG contained no noise or abnormalities in muscle motion, respiratory variability, or baseline wandering, and was within the expected peak amplitude range. There is no noise in the Q waveform detected; therefore the heart rate is correctly detected. T-wave has been filtered from 0.05 to $0.07 \mathrm{mV}$ as expected. The heart rate of the analyzed signal ranges between 79 and 82 BPM. Consequently, the validity of the algorithm used to build simulation blocks for cardiac surveillance has been proven.

\section{REFERENCES}

[1] Malek, A. S., Elnahrawy, A., Anwar, H., \& Naeem, M. (2020) Automated detection of premature ventricular contraction in ECG signals using enhanced template matching algorithm. Biomedical Physics \& Engineering Express, 6(1), 015024.

[2] Rueda, C., Larriba, Y., \& Lamela, A. (2021). The hidden waves in the ECG uncovered revealing a sound automated interpretation method. Scientific reports, 11(1), 1-11.

[3] Chatterjee, S., Thakur, R. S., Yadav, R. N., Gupta, L., \& Raghuvanshi, D. K. (2020). Review of noise removal techniques in ECG signals. IET Signal Processing, 14(9), 569-590.

[4] Fotiadou, E., Konopczyński, T., Hesser, J., \&Vullings, R. (2020). Endto-end trained encoder-decoder convolutional neural network for fetal electrocardiogram signal denoising. Physiological measurement, 41(1), 015005.

[5] Xue, J., \& Yu, L. (2021). Applications of Machine Learning in Ambulatory ECG. Hearts, 2(4), 472-494.

[6] Etzkorn, L. H., Heravi, A. S., Wu, K. C., Post, W. S., Urbanek, J., \&Crainiceanu, C. (2021). Classification of Free-Living Body Posture with ECG Patch Accelerometers: Application to the Multicenter AIDS Cohort Study. bioRxiv.

[7] Atanasov, V., Sivkov, Y., \&Velikov, N. (2020, September). An approach of Feature extraction of ECG signal of CLAS database. In 2020 International Conference on Biomedical Innovations and Applications (BIA) (pp. 93-96). IEEE.

[8] Latchoumi, T. P., Balamurugan, K., Dinesh, K., \& Ezhilarasi, T. P. (2019). Particle swarm optimization approach for waterjet cavitation peening. Measurement, 141, 184-189.

[9] Zhang, S., Zhao, T., Peng, C., Li, Q., \& Zhang, X. (2020, June). Design and Implementation of A Novel Real-Time P-QRS-T Waves Detection Algorithm. In 2020 IEEE 4th Information Technology, Networking, Electronic and Automation Control Conference (ITNEC) (Vol. 1, pp. 1109-1112). IEEE.

[10] Kashou, A. H., Ko, W. Y., Attia, Z. I., Cohen, M. S., Friedman, P. A., \& Noseworthy, P. A. (2020). A comprehensive artificial intelligenceenabled electrocardiogram interpretation program. Cardiovascular Digital Health Journal, 1(2), 62-70.

[11] Pilia, N., Nagel, C., Lenis, G., Becker, S., Dössel, O., \& Loewe, A. (2021). ECGdeli-an open source ecg delineation toolbox for MATLAB. SoftwareX, 13, 100639.

[12] Zhang, Q., \& Li, X. (2021, April). Analysis and Application of Bayesian Network and Qt View Framework in Network Fault Location. In 2021 6th International Conference on Intelligent Computing and Signal Processing (ICSP) (pp. 981-984). IEEE.

[13] Garikapati, P., Balamurugan, K., Latchoumi, T. P., \&Malkapuram, R. (2021). A Cluster-Profile Comparative Study on Machining AlSi 7/63\% of SiC Hybrid Composite Using Agglomerative Hierarchical Clustering and K-Means. Silicon, 13, 961-972.

[14] Wang, Y., Wu, L., Chen, C., Jin, Z., Li, Z., \& Wang, Y. (2020, August). $\mathrm{P}$ wave Detection in Electrocardiogram Based on Wavelet Transform and Differential Correction. In 2020 12th International Conference on Advanced Computational Intelligence (ICACI) (pp. 277-282). IEEE.

[15] Sun, J. Y., Shen, H., Qu, Q., Sun, W., \& Kong, X. Q. (2021). The application of deep learning in electrocardiogram: Where we came from and where we should go? International journal of cardiology.

[16] Ezhilarasi, T. P., Dilip, G., Latchoumi, T. P., \& Balamurugan, K. (2020). UIP-A Smart Web Application to Manage Network Environments. In Proceedings of the Third International Conference on Computational Intelligence and Informatics (pp. 97-108). Springer, Singapore.

[17] Ohmuta, T., Mitsui, K., \& Shibata, N. (2020). ECG QT-Interval measurement using wavelet transformation. Sensors, 20(16), 4578.

[18] Attia, Z. I., Harmon, D. M., Behr, E. R., \& Friedman, P. A. (2021). Application of artificial intelligence to the electrocardiogram. European Heart Journal, 42(46), 4717-4730.

[19] Surekha, K. S., \& Patil, B. P. (2021). Power line interference removal from electrocardiogram signal using multi-order adaptive LMS filtering. International Journal of Biomedical Engineering and Technology, 35(2), 135-151.

[20] Andršová, I., Hnatkova, K., Šišáková, M., Toman, O., Smetana, P., Huster, K. M., ... \& Malik, M. (2021). Influence of heart rate correction formulas on QTc interval stability. Scientific Reports, 11(1), 1-21.

[21] Kumar, A., Ranganatham, R., Singh, S., Komaragiri, R., \& Kumar, M. (2021). A robust digital ECG signal watermarking and compression using biorthogonal wavelet transform. Research on Biomedical Engineering, 37(1), 79-85.

[22] Guven, G., Guz, U., \& Gürkan, H. (2022). A novel biometric identification system based on fingertip electrocardiogram and speech signals. Digital Signal Processing, 121, 103306. 